\title{
Spin Supercurrent in Phenomena of Quantum Non-Locality (Quantum Correlations, Magnetic Vector Potential) and in Near-Field Antenna Effect
}

\author{
Liudmila B. Boldyreva \\ The State University of Management, Moscow, Russia \\ Email: boldyrev-m@yandex.ru
}

How to cite this paper: Boldyreva, L.B. (2019) Spin Supercurrent in Phenomena of Quantum Non-Locality (Quantum Correlations, Magnetic Vector Potential) and in Near-Field Antenna Effect. Journal of Modern Physics, 10, 128-144.

https://doi.org/10.4236/jmp.2019.102010

Received: December 31, 2018

Accepted: February 23, 2019

Published: February 26, 2019

Copyright () 2019 by author(s) and Scientific Research Publishing Inc. This work is licensed under the Creative Commons Attribution International License (CC BY 4.0).

http://creativecommons.org/licenses/by/4.0/

(c) (i) Open Access

\begin{abstract}
It is shown that such phenomena as quantum correlations (interaction of space-separated quantum entities), the action of magnetic vector potential on quantum entities in the absence of magnetic field, and near-field antenna effect (the existence of superluminally propagating electromagnetic fields) may be explained by action of spin supercurrents. In case of quantum correlations between quantum entities, spin supercurrent emerges between virtual particles pairs (virtual photons) created by those quantum entities. The explanation of magnetic vector potential and near-field antenna effect is based on contemporary principle of quantum mechanics: the physical vacuum is not an empty space but the ground state of the field consisting of quantum harmonic oscillators (QHOs) characterized by zero-point energy. Using the properties of the oscillators and spin supercurrent, it is proved that magnetic vector potential is proportional to the moment causing the orientation of spin of QHO along the direction of magnetic field. The near-field antenna effect is supposed to take place as a result of action of spin supercurrent causing secondary electromagnetic oscillations. In this way, the electromagnetic field may spread at the speed of spin supercurrent. As spin supercurrent is an inertia free process, its speed may be greater than that of light, which does not contradict postulates of special relativity that sets limits to the speed of inertial systems only.
\end{abstract}

\section{Keywords}

Spin Supercurrent, Quantum Correlations, Magnetic Vector Potential, Near-Field Antenna Effect, Zero-Point Energy, Quantum Harmonic Oscillator, Virtual Particles Pair, Virtual Photon 


\section{Introduction}

The following phenomena are discussed in this work: 1) quantum correlations-mutual dependence of characteristics of wave function of so-called entangled quantum entities while there is space separation; 2) a change in characteristics of wave function of quantum entities while they are passing in the region of non-zero magnetic vector potential (magnetic field may be absent); 3) the near-field antenna effect-the existence near antenna (an oscillating electric dipole) of superluminally propagating electromagnetic field. The common property of the phenomena is the following: they are not described by action of well-known physical fields: electric, magnetic, gravitational. For description of these phenomena, the mathematical apparatus of quantum mechanics is used: Heisenberg's uncertainty principle, Schrödinger equation for wave function of quantum entities [1] [2] [3].

Let us consider these phenomena in detail.

Quantum correlations belong to the category of phenomena that are collectively called "quantum non-locality" [4] [5]. The essence of the phenomena can be described using the following example. Let two quantum entities (Figure 1) a and $\boldsymbol{b}$ emitted by the same source and having the same wave function at the initial moment of time move in different directions.

Entity $\boldsymbol{a}$ is directed, depending on the position ( 1 or 2 ) of switch $P$, either towards detector $A_{1}$ or detector $A_{2}$ (these detectors have different characteristics); entity $\boldsymbol{b}$ is directed towards detector $B$. According to postulates of quantum mechanics, the wave properties of entity $\boldsymbol{b}$ being detected will depend on that which detector detects $\boldsymbol{a}$. In the early years of studies of quantum correlations, it was supposed that these correlations take place only between "entangled" quantum entities emitted by one source and described by a single wave function [5]. However, the discovery of quantum correlation between the photons having equal frequencies, emitted by different sources [4], suggests that for the existence of quantum correlations, it is only necessary for the interacting quantum entities to have equal wave function frequencies and at least for photons to have a definite mutual spin polarization. Quantum correlations have a non-electric and non-magnetic nature and take place independent of the distance between the interacting quantum entities [5] [6]. The speed of quantum correlations is greater than the speed of light; it follows from the possibility of correlations of photons separated in space and simultaneously emitted. The experiments exist [7] in which it is shown that the speed of quantum correlations is $10^{4}$ times greater than the speed of light. If quantum correlations are accomplished by an inertia-free process (it is not accompanied by emergence of mass), this does not contradict the postulates of special relativity that set limits to the speed of motion of inertial systems only [8].

It should be noted that Einstein, Podolsky and Rosen with reference to the measurement problem of entangled quantum entities wrote [9]: “... the description of reality given by the wave function in quantum mechanics is not complete", which in fact admits a possibility of existence of a physical process re- 
sponsible for quantum correlations.

Field-free magnetic vector potential. In classical electrodynamics, the magnetic field of induction $\mathbf{B}$ is determined [10] by equation $\mathbf{B}=\operatorname{curl} \mathbf{A}$, where $\mathbf{A}$ is a magnetic vector potential. In shielding of magnetic field, $\mathbf{B}=0$, the following may take place: $\mathbf{A} \neq 0$. This case is referred to as the field-free vector potential. Magnetic vector potential has a physical meaning of its own. In 1949, Erenberg and Siday predicted the ability of magnetic vector potential to influence directly the characteristics of quantum entities even though there is no electromagnetic field at the location of the entities [11]. In 1959, the possibility of such an effect was considered by Aharonov and Bohm [12]. Subsequently, a great number of experiments have been conducted which confirmed the theoretical predictions [13]. In general, these experiments were as follows (see Figure 2): the beam of electrically charged quantum entities emitted by a source is split into two beams: $C_{1}$ and $C_{2}$. Beam $C_{1}$ propagates through the region where $\mathbf{A}=0$. Beam $C_{2}$ passes through an energized toroidal solenoid (region $\delta$ ). The solenoid is shielded in such a way that outside the solenoid there is no magnetic field, $\mathbf{B}=0$, but the vector potential is present: $\mathbf{A} \neq 0$. Both beams of quantum entities arrive at the entrances of an interferometer. The interference rings obtained suggest that there is a change in the wave function phase of quantum entities passing through the region where $\mathbf{B}=0$ and $\mathbf{A} \neq 0$.

In quantum mechanics, the description of action of the field-free magnetic vector potential is based on Schrödinger's equation [2] without introducing any physical process. As the action of the field-free magnetic vector potential takes place in space where electromagnetic field is absent, this potential has both non-electric and non-magnetic nature.

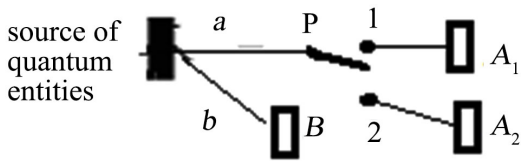

Figure 1. Schematic diagram of the experiment that illustrates quantum correlations between quantum entities. $a$ and $b$ are quantum entities; $A_{1}, A_{2}$, and $B$ are detectors; $P$ is a switch with positions 1 and 2 .

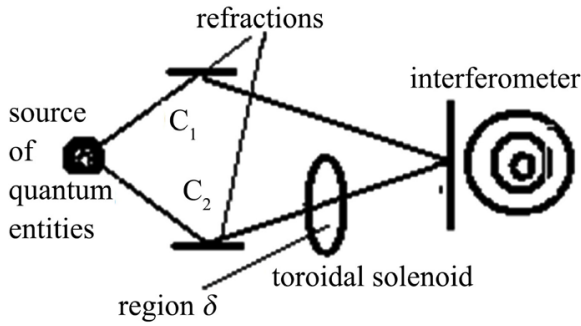

Figure 2. Schematic diagram of the experiment on the study of the effects of magnetic vector potential on quantum entities. The source of quantum entities emits two beams. Beam $C_{1}$ propagates through the region where $\mathbf{A}=0$. Beam $C_{2}$ passes through the shielded energized toroidal solenoid in the region (region $\delta$ ) of the field-free magnetic vector potential. Interference rings are produced by the interferometer. 
Near-field antenna effect. There is experimental evidence of existence near antenna (an oscillating electric dipole) of superluminally propagating electromagnetic field [14] [15]. The well-known explanation of this phenomenon [14] is based on the assumption that a superluminal motion takes place at distances of about one wavelength from the source of oscillations. The wavelength of wave function of quantum entities (for photon it is equal to photon's wavelength) is the size on which the Heisenberg's uncertainty principle [1] holds true. That is the uncertainty $\Delta p_{x}$ in the measured value of component $p_{x}$ of momentum $\mathbf{p}$ of quantum entity is determined as: $\Delta p_{x} \geq \hbar / \Delta x$ ( $\Delta x$ is the uncertainty in the measured value of coordinate $x$ of quantum entity). As momentum is a function of speed of quantum entity, the uncertainty in measured value of momentum means the uncertainty in the value of speed of quantum entity. Thus the suggested explanation of near-field antenna effect is in accordance with the second postulate of special relativity asserting the constancy of the velocity of light.

This work suggests essentially a new approach to description of the above-considered phenomena: namely it is shown that it is possible to describe these phenomena in terms of such physical process as spin supercurrent. The spin supercurrent emerges between objects having spin, and its action tends to make equal the respective characteristics of precession of spins of interacting objects. (Note that Yuri Bunkov, Vladimir Dmitriev and Igor Fomin were awarded the Fritz London Memorial Prize in 2008 for the studies of spin supercurrents in superfluid ${ }^{3} \mathrm{He}-\mathrm{B}$ [16] [17] [18]). The specific feature of the approach to description of the above-considered phenomena is as well that it takes into account the properties of the physical vacuum.

In quantum field theory, the physical vacuum, free from magnetic and electric fields (without regard to gravitational energy), is defined not as an empty space but as the ground state of the field consisting of quantum harmonic oscillators (called QHOs from now on) characterized by zero-point energy [19]. The concept of zero-point energy was developed in Germany in 1913 by a group of physicists, including M. Planck, A. Einstein, and O. Stern [20]. A quantum entity (its characteristics are determined by the wave function) which is a singularity in electric or magnetic fields (electric charge or/and magnetic dipole) creates a virtual photon (virtual particles pair) having spin in the physical vacuum [21].

It is proved in this work that the properties of quantum correlations, the action of magnetic vector potential on quantum entities, and near-field antenna effect are determined by the properties of spin supercurrent arising between virtual photons created by quantum entities participating in these phenomena and of spin supercurrents emerging between virtual photons created by the quantum entities, on the one hand, and QHOs that constitute the physical vacuum, on the other hand.

The findings of this work can be used, for example, for optimization of channels intended for performing quantum correlations between quantum entities 
owing to increasing the number of characteristics by which the correlation of the quantum entities is effected.

The paper below consists of the following sections. Section 2 (titled as "The Characteristics of Spin Supercurrent") contains equations describing the conditions of emergence of spin supercurrent and effects of the latter, energy properties of the current and features of its propagation (the speed, the impossibility of shielding by electromagnetic screens). Section 3 (titled as "The Properties of QHOs”) contains the description of such characteristics of QHO as the electric dipole moment, mass, precessing spin and connection of QHO speed with magnetic phenomena. Section 4 (titled as "Results") provides explanations of phenomena of quantum correlations, the action of magnetic vector potential on quantum entities, and near-field antenna effect as based on both the properties of spin supercurrent and those of the physical vacuum consisting of QHOs.

Below are specified the main variables used in this paper.

The characteristics of spin supercurrent: $\alpha_{1}$ and $\alpha_{2}$ are angles of precession, $\theta_{1}$ and $\theta_{2}$ are angles of deflection, $\omega_{1}$ and $\omega_{2}$ are frequencies of precession of spins, $J_{z}$ is spin supercurrent.

The characteristics of QHO: $\mathbf{S}_{Q H O}$ is spin, $m_{Q H O}$ is mass, $\boldsymbol{\Omega}_{\mathrm{QHO}}$ is precession frequency, $\mathbf{d}_{Q H O}$ is electric dipole moment, $\mathbf{u}$ is velocity of $\mathrm{QHO}, \mathbf{A}$ is magnetic vector potential, $\mathbf{E}_{Q H O}$ is electric field inside the QHO, $\mathbf{M}_{Q H O}$ is the moment causing precession of spin $\mathbf{S}_{Q H O}$.

\section{The Characteristics of Spin Supercurrent}

1) The spin supercurrent arises between objects with precessing spins (spin structures) [16] [17] [18]. Figure 3 contains the schema of such spin structures with the following characteristics: $\alpha_{1}$ and $\alpha_{2}$ are precession angles, $\theta_{1}$ and $\theta_{2}$ are deflection angles, $\omega_{1}$ and $\omega_{2}$ are the frequencies of precession of spins $\mathbf{S}$ of the structures, $J_{z}$ is spin supercurrent between the spin structures.

2) The spin supercurrent tends to equalize the respective characteristics of spins of interacting spin structures: angles (phases) of precession and angles of deflection. For example, the value of spin supercurrent $J_{z}$ in the direction of orientation (axis $\boldsymbol{z}$ in Figure 3 ) of precession frequencies of spins is determined as follows:

$$
J_{z}=g_{1}\left(a_{1}-\alpha_{2}\right)+g_{2}\left(\theta_{1}-\theta_{2}\right) \text {, }
$$

where $g_{1}$ and $g_{2}$ are coefficients depending on deflection angles and the properties of the medium where spin supercurrent emerges. Let us assume that before the action of spin supercurrent the angles of precession $\alpha_{1}$ and $\alpha_{2}$ are determined as $\alpha_{1}=\omega_{1} t+\alpha_{01}$ and $\alpha_{2}=\omega_{2} t+\alpha_{02}$, where $\alpha_{01}$ and $\alpha_{02}$ are the values of angles of precession respectively $\alpha_{1}$ and $\alpha_{2}$ at $t=0$. If $\alpha_{01}=\alpha_{02}=0$, then

$$
\begin{aligned}
& \alpha_{1}=\omega_{1} t, \\
& \alpha_{2}=\omega_{2} t .
\end{aligned}
$$




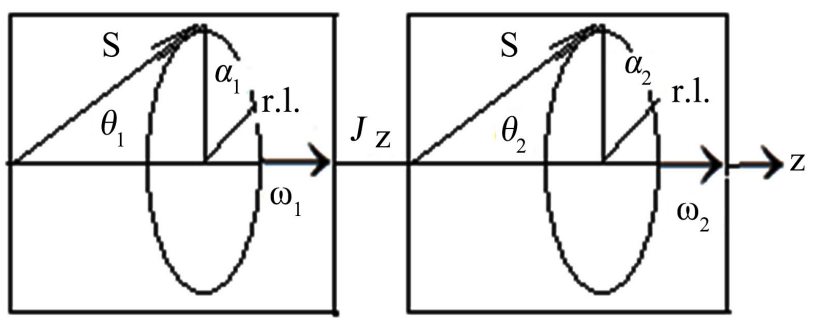

Figure 3. The schema of spin structures. $J_{z}$ is spin supercurrent between spin structures with the following characteristics: $\alpha_{1}$ and $\alpha_{2}$ are precession angles, $\theta_{1}$ and $\theta_{2}$ are deflection angles, $\omega_{1}$ and $\omega_{2}$ are precession frequencies oriented along axis $\mathrm{z}$, $\mathrm{S}$ is spin, r.l. is a reference line.

As a result of the action of spin supercurrent at arbitrary moment $t$, according to definition of this current, the following holds: $\left|\theta_{1}-\theta_{2}\right|>\left|\theta_{1}^{\prime}-\theta_{2}^{\prime}\right|$ and:

$$
\left|\alpha_{1}-\alpha_{2}\right|>\left|\alpha_{1}^{\prime}-\alpha_{2}^{\prime}\right|
$$

where: $\theta_{1}^{\prime}$ and $\theta_{2}^{\prime}$ are respectively the values of deflection angles $\theta_{1}$ and $\theta_{2}$ of precessing spins of interacting spin structures after the action of spin supercurrent; $\alpha_{1}^{\prime}$ and $\alpha_{2}^{\prime}$ are respectively the values of precession angles $\alpha_{1}$ and $\alpha_{2}$ of precessing spins of interacting spin structures after the action of spin supercurrent. As a result of changes in precession angles, the change in frequencies $\omega_{1}$ and $\omega_{1}$ of interacting spin structures may also take place:

$$
\left|\omega_{1}-\omega_{2}\right|>\left|\omega_{1}^{\prime}-\omega_{2}^{\prime}\right| \text {, }
$$

where $\omega_{1}^{\prime}$ and $\omega_{2}^{\prime}$ are the values of spin structures precession frequencies $\omega_{1}$ and $\omega_{1}$ after the action of spin supercurrent.

3) At a definite nonzero difference in the values of angles of precession

$$
\Delta \alpha_{c}=\alpha_{1}-\alpha_{2} \neq 0,
$$

a phase slippage, that is, the drop in the value and change in the sign of spin supercurrent may take place. In this case, Equation (1) does not hold. Consequently, as follows from Equations (2)-(3) and (6), for the effective equalization of respective characteristics of interacting spin structures the following is necessary: 1) a definite mutual orientation of precession frequencies of the interacting spin structures; 2) the absence of phase slippage, in particular, this takes place if the difference between their precession frequencies, $\omega_{1}-\omega_{2}$, satisfy the following condition:

$$
\Delta \omega \rightarrow 0
$$

4) The effectivity of action of spin supercurrent between spin structures does not depend on the distance between them. For example, the action of spin supercurrent in superfluid ${ }^{3} \mathrm{He}-\mathrm{B}$ is limited only by the volume of superfluid.

5) Spin supercurrent is not an electric or magnetic process and consequently it is not shielded by electromagnetic screens.

6) The changes in the precession and deflection angles of precessing spins of interacting spin structures due to the action of spin supercurrent may be 
considered as spin orientation transfer in the physical vacuum. Through the example of photon it may be shown that the spin orientation transfer in the physical vacuum is a dissipation-free process.

According to [22], the photon spin $\mathbf{S}_{p h}$ is perpendicular to its velocity $\mathbf{c}$, $\mathbf{S}_{p h} \perp \mathbf{c}$, and in the pure state (circular polarization) the photon spin performs precession motion with frequency $\boldsymbol{\omega}_{p h}, \boldsymbol{\omega}_{p h} \uparrow \uparrow \mathbf{c}$. According to [23], the energy $W_{\text {prec }}$ of precession with frequency $\boldsymbol{\omega}_{p h}$ of angular momentum $S_{p h}=\hbar$ at $\mathbf{S}_{p h} \perp \boldsymbol{\omega}_{p h}$ is determined as:

$$
W_{\text {prec }}=\hbar \omega_{p h} .
$$

Energy $U_{p h}$ of photon must be the sum of two components: energy $W_{\text {prec }}$ of precession motion of spin and energy $W_{S}$ of spin orientation transfer in space. From experiments [3] it follows that the total energy of photon is equal to $\hbar \omega_{p h}$. Taking into account Equation (8), this means that the total energy of photon $U_{p h}$ equals only energy $W_{\text {prec }}$ of precession motion of spin and the energy of spin orientation transfer equals zero:

$$
W_{S}=0 \text {. }
$$

Due to spreading of spin supercurrent without dissipation, the energies of interacting spin structures as a result of action of this current change by the same value. As, according to Equation (8), the energy of precessing spin is proportional to the frequency of precession, then from condition (5) it follows that $\omega_{1}-\omega_{1}^{\prime}=-\left(\omega_{2}-\omega_{2}^{\prime}\right)$ or:

$$
\Delta \omega_{1}=-\Delta \omega_{2},
$$

where $\Delta \omega_{1}=\omega_{1}-\omega_{1}^{\prime}$ and $\Delta \omega_{2}=\omega_{2}-\omega_{2}^{\prime}$. Then according to Equations (2)-(4), $\alpha_{1}-\alpha_{1}^{\prime}=-\left(\alpha_{2}-\alpha_{2}^{\prime}\right)$ or:

$$
\Delta \alpha_{1}=-\Delta \alpha_{2},
$$

where $\Delta \alpha_{1}=\alpha_{1}-\alpha_{1}^{\prime}$ and $\Delta \alpha_{2}=\alpha_{2}-\alpha_{2}^{\prime}$.

7) As energy is intrinsically associated with mass, then, according to Equation (9), spin supercurrent performing spin orientation transfer is not accompanied by emergence of any mass, that is, it is an inertia free process and consequently the speed $y_{s s}$ of spin supercurrent may be greater than the speed of light:

$$
y_{s s}>c \text {. }
$$

This does not contradict the postulates of special relativity which set limits to the speed of inertial systems only [8].

\section{The Properties of QHOs}

According to contemporary principle of quantum mechanics, the physical vacuum is defined not as an empty space but as the ground state of the field consisting of QHOs characterized by zero-point energy. Let us consider the properties of QHO in detail (see also [19] [24] [25]).

1) The energy of QHO is equal to $\hbar \Omega_{Q H O} / 2$, the energy is referred to as zero-point energy. This expression for energy of QHO coincides with the expres- 
sion for energy of object having angular momentum $\hbar$ and performing oscillations with frequency $\boldsymbol{\Omega}_{\mathrm{OHO}} \quad$ [26].

2) The QHO has mass $m_{\mathrm{QHO}}$ associated with the energy of QHO.

3) The existence of electric polarization of physical vacuum [3] suggests that QHO is an electric dipole (let us denote the electric dipole moment as $\mathbf{d}_{O H O}$ ).

4) There are some phenomena which testify that QHOs constituting the physical vacuum must have precessing spin for example: 1) the Cherenkov effect [27], the production of photons having spin by an electron moving at a superluminal speed while saving the value of its own spin; 2) the creation of virtual photons having precessing spin [21] by a quantum entity while saving the value of its own spin. If the principle of conservation of angular momentum holds true in the physical vacuum, then spin of virtual photon consists of spins of QHOs that constitute this vacuum.

That is, it may be supposed that QHO has spin $\mathbf{S}_{Q H O}$ and the frequency of oscillations $\boldsymbol{\Omega}_{\mathrm{OHO}}$ is the precession frequency of $\mathbf{S}_{\mathrm{QHO}}$. That is, QHO as well as a virtual photon is a spin vortex.

5) Since QHO is a spin vortex, then for it as well as for analogous characteristics (spin, electric dipole moment, the precession frequency of spin, the deflection angle) of other spin vortices (photon, virtual photon)) the following takes place [24] [25] [28].

$$
\mathbf{d}_{\mathrm{QHO}} \uparrow \downarrow \mathbf{S}_{\mathrm{QHO}} .
$$

As electric field $\mathbf{E}_{\text {ОНО }}$ inside the electric dipole is antiparallel to its electric dipole moment, i.e. $\mathbf{E}_{\mathrm{OHO}} \uparrow \downarrow \mathbf{d}_{\mathrm{OHO}}$ [10], from condition (13) we have:

$$
\begin{gathered}
\mathbf{E}_{\mathrm{OHO}} \uparrow \uparrow \mathbf{S}_{\mathrm{QHO}} . \\
\boldsymbol{\Omega}_{\mathrm{OHO}} \| \mathbf{u},
\end{gathered}
$$

where $u$ is the speed of $\mathrm{QHO}$,

$$
\sin \theta=u / c,
$$

where $\theta$ is the angle between $\mathbf{S}_{\mathrm{QHO}}$ and $\boldsymbol{\Omega}_{\mathrm{OHO}}$ (angle of deflection), $c$ is the speed of light.

6) That $\mathrm{QHO}$ has an electric dipole moment means that there is a repulsive force inside the $\mathrm{QHO}$ balancing the attractive Coulomb force between oppositely charged parts inside the QHO. The existence of such repulsive force may be treated as the existence of omniradial tensions inside the QHO.

7) It is shown in [26] that there is a complete analogy between the structures of formulas describing the magnetic interactions of current-carrying wires and the structures of formulas describing the interactions of vortices in an ideal incompressible liquid with positive density and negative pressure. The above considered properties of QHOs suggest that the physical vacuum consisting of QHOs is similar to such a liquid. The density $\rho$ of this vacuum is formed by mass $m_{\text {QHO }}$ of QHO; negative pressure $p$ is a result of omniradial tensions inside the QHO. Hydrodynamically, the stationary motion of this vacuum as a 
continuum may be described in the absence of shear viscosity as:

$$
\rho u^{2} / 2-p=\text { const } \text {. }
$$

Let us deduce the relationship between the speed of QHOs and magnetic induction by comparing the characteristics of the magnetic field and both force and kinematic characteristics of the medium described by Equation (17) [24] [25] [29]. These equations are written, first, for the vacuum whose permeability equals 1, and, secondly, they are written in the CGSE system of units, so that the equations include constant $c$ which is a characteristic of the medium whose motion results in magnetic phenomena [10].

The magnetic induction B generated by a loop with current $I[10]$ is determined by the Biot-Savart law and in the CGSE system of units defined as $\mathbf{B}=\frac{I}{c} \int_{L^{\prime}} \frac{\mathrm{d} \mathbf{l} \times \mathbf{r}}{r^{3}}$, where $L^{\prime}$ is the length of the loop, $\mathrm{d} \mathbf{l}$ is the wire element, $\mathbf{r}$ is a radius vector from $\mathrm{d} \mathbf{l}$ to the point of observation. The field of velocities $\mathbf{u}$ generated by a closed vortex line having circulation $\Gamma$ along an arbitrary loop enclosing the vortex line is defined [26] as $\mathbf{u}=\frac{\Gamma}{4 \pi} \int_{L^{\prime}} \frac{\mathrm{d} \mathbf{l} \times \mathbf{r}}{r^{3}}$, where $\mathrm{dl}$ is an infinitesimal vector element of the vortex line, $L^{\prime}$ is the length of the line. Equating the expressions for $\mathbf{B}$ and $\mathbf{u}$, we obtain the relationship between $\Gamma$ and I:

$$
\Gamma=\mathbf{I} \sqrt{4 \pi} /(c \sqrt{\rho})
$$

Note. As shown in [24] [25], the electric current I creates the circulation $\Gamma$ in the physical vacuum consisting of QHOs due to precession motion of spins of virtual photons (virtual particles pairs) that are created by moving charged quantum entities in the current-carrying wire.

The force $F_{\Gamma}$ acting on a unit length of either of the two infinite mutually parallel vortex lines having the same values of circulation $\Gamma$ equals: $F_{\Gamma}=\rho \Gamma^{2} /\left(2 \pi r_{w}\right)$, where $r_{w}$ is the distance between the vortex lines with circulation $\Gamma$ [26]. The force $F_{I}$ acting on a unit length of either of the two infinite mutually parallel current-carrying wires having the same values of current I equals: $F_{I}=2 I^{2} /\left(r_{w} c^{2}\right)$, where $r_{w}$ is here the distance between the current-carrying wires [10]. Equating the expressions for $F_{\Gamma}$ and $F_{I}$ and taking into account Equation (18), we obtain.

$$
\mathbf{B}=\mathbf{u} \sqrt{4 \pi \rho} .
$$

Thus the motion of physical vacuum consisting of QHOs characterized by zero-point energy is a cause of magnetic phenomena.

The QHO characteristics considered in this section are given in Figure 4: spin $\mathbf{S}_{\mathrm{OHO}}$, frequency of the spin precession $\boldsymbol{\Omega}_{\mathrm{OHO}}$, electric dipole moment $\mathbf{d}_{\mathrm{OHO}}$, mass $m_{Q H O}, \mathrm{QHO}$ velocity $\mathbf{u}$, electric field inside the QHO $\mathbf{E}_{\mathrm{QHO}}$, angle of deflection $\theta$, magnetic vector potential $\mathbf{A}, \mathbf{M}_{\mathrm{QHO}}$ is the moment causing precession of spin. The latter two characteristics are considered in Section 4. 


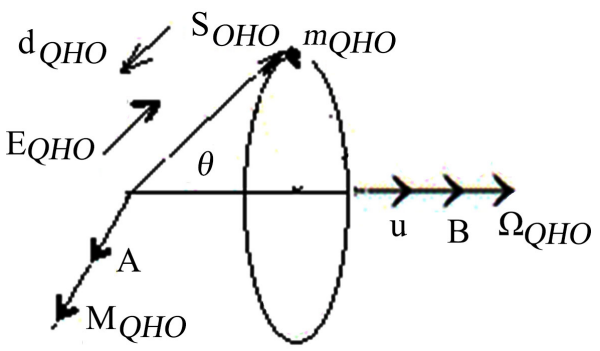

Figure 4. The characteristics of a quantum harmonic oscillator (QHO): $\mathbf{S}_{Q H O}$ is spin, $m_{\text {OHO }}$ is the mass, on the assumption that it is a point-like mass, $\boldsymbol{\Omega}_{\mathrm{OHO}}$ is the precession frequency, $\mathbf{d}_{\text {Qно }}$ is the electric dipole moment, $\mathbf{u}$ is the velocity of QHO, $\mathbf{A}$ is the magnetic vector potential, $\theta$ is the angle of deflection, $\mathbf{E}_{\mathrm{OHO}}$ is the electric field inside the $\mathrm{QHO}, \mathbf{M}_{\mathrm{QHO}}$ is the moment causing precession of spin $\mathbf{S}_{\mathrm{QHO}}, \mathbf{B}$ is magnetic induction.

\section{Results}

\subsection{Quantum Correlations}

Let us prove that the properties of quantum correlations considered in Introduction are in accordance with the properties of spin supercurrent (see also the study by L. Boldyreva [30]).

1) The quantum correlation takes place between quantum entities having equal frequencies of their wave functions. As follows from [30], the precession frequency $\omega_{v}$ of spin of the virtual photon (pair of virtual particles) created by the quantum entity equals the frequency $\omega_{q}$ of wave function of the entity:

$$
\omega_{q}=\omega_{v} .
$$

Consequently, the first property of quantum correlation is in accordance with condition (7) of effective action of spin supercurrent: the equality of the precession frequencies of interacting spin structures.

2) The quantum correlation between photons takes place at definite mutual polarization $\left(\mathbf{E}_{p h}\right)$ of photons. Due to transverse orientation of photon spin $\mathbf{S}_{p h}\left(\mathbf{S}_{p h} \perp \mathbf{c}\right)$ it holds that $\mathbf{S}_{p h} \uparrow \uparrow \mathbf{E}_{p h}$. Thus the quantum correlation between photons takes place at definite mutual orientation of spins of photons. If to take into account that photon's frequency $\omega_{p h}$ is oriented along $\mathbf{c}, \omega_{p h} \uparrow \uparrow \mathbf{c}$, then $\mathbf{S}_{p h} \perp \boldsymbol{\omega}_{p h}$. Consequently, quantum correlation takes place at definite mutual orientation of photons' frequencies. This property is in accordance with the definition of spin supercurrent, see Equation (1), according to which the orientation of spin supercurrent relates to the orientation of the precession frequencies of spins of interacting spin structures.

3) The quantum correlations take place independent of the distance between the interacting quantum entities. This property agrees with property 4 of spin supercurrent.

4) The quantum correlations have non-electric and non-magnetic nature [4] [5]. This property is in accordance with property 5 of spin supercurrent.

5) The speed of quantum correlations is greater than the speed of light. As it 
follows from experiments [7], the speed of quantum correlations is $10^{4}$ times greater than the speed of light. This property is in accord with condition (12) (property 7 of spin supercurrent).

6) The relation between the changes in phases and frequencies of waves in the two-photon interference, associated with quantum correlations, is analogous to the changes in phases (angles of precession) and frequencies of spin precession in spin structures under action of spin supercurrent. Figure 5 shows a diagram of experimental setup, which illustrates two-photon interference provided both beams of photons undergo phase delays [4].

Fields $\gamma_{1}$ and $\gamma_{2}$ of frequency $\omega_{\gamma}$ and also fields $\xi_{1}$ and $\xi_{2}$ of frequency $\omega_{\xi}$ are mixed by respective beam splitters. The detectors and coincidence circuit measure the correlation of intensities of output fields $\gamma$ and $\xi$, the correlation being dependent on phase delays $\vartheta_{1}$ and $\vartheta_{2}$.

Depending on the statistics of incident beams of light, two types of interference may take place: one with phase $\vartheta_{1}-\vartheta_{2}$, the other with phase $\vartheta_{1}+\vartheta_{2}$. The first interference is referred to as the Hanbury Brown and Twiss intensity interference, the second one is called the two-photon interference. Under definite conditions, both types of interference are a result of conversion by beam splitters of fluctuations of phases of input fields into fluctuations of intensities of output fields [4]. Let us consider these conditions. Let fields $\gamma_{k}$ and $\xi_{k}(k=1,2)$ have constant unit amplitudes and phases $x_{k}(t)$ and $z_{k}(t)$ drifting in time $t$. That is the following takes place: $\gamma_{k}(t)=\exp \left[-i x_{k}(t)\right], \quad \xi_{k}(t)=\exp \left[-i z_{k}(t)\right]$. Then the first condition for the ordinary interference of intensities is determined by equalities:

$$
x_{1}=z_{1}, \quad x_{2}=z_{2} .
$$

The second condition is as follows:

$$
x_{1}+z_{1}=x_{2}+z_{2},
$$

that is, fluctuations of light beam phases occur in such a way that the sum of the phases remains constant.

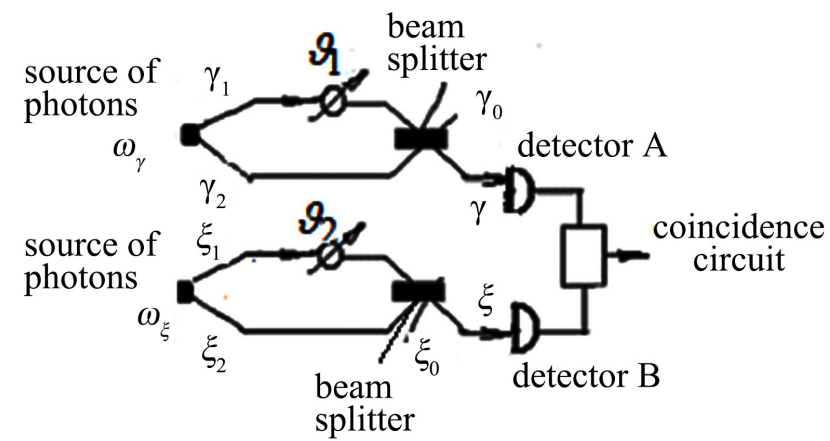

Figure 5. Diagram of a four-mode intensity interferometer. $\gamma_{1}$ and $\gamma_{2}, \xi_{1}$ and $\xi_{2}$ are input beams of light; $\vartheta_{1}$ and $\vartheta_{2}$ are phases of delay of light beams; $\omega_{\gamma}$ is the frequency of output beam $\gamma ; \omega_{\xi}$ is the frequency of output beam $\xi ; \gamma_{0}$ and $\xi_{0}$ may be classified as idle modes of respective beams. The detectors and coincidence circuit measure the correlation of intensities. 
Equalities (21) can be called the conditions of "classical" correlation; it may be associated with thermal fluctuations; condition (22) is a condition of anticorrelation of phases which is a quantum phenomenon (related with quantum correlations [4]). From condition (22) it follows that for the changes in the phase of field $\gamma, \Delta x$, and field $\xi, \Delta z$, the following is valid:

$$
\Delta x=-\Delta z .
$$

Equality (23) is similar to Equation (11) describing the property of spin supercurrent (property 6 of spin supercurrent).

The frequencies of beams in the experiment on the two-photon interference as well as the phases drift in opposite directions, which is in accordance with condition (10) of property 6 of spin supercurrent.

Thus all properties of quantum correlations are in accordance with the properties of spin supercurrents.

\subsection{Magnetic Vector Potential}

According to condition (15) and Equation (19), in magnetic field B two types of motion of $\mathrm{QHO}$ that constitute physical vacuum take place: the translational motion with velocity $\mathbf{u}=\mathbf{B} / \sqrt{4 \pi \rho}$ and precession of the $\mathrm{QHO}$ spin with frequency $\boldsymbol{\Omega}_{\mathrm{OHO}}$. The value of $\boldsymbol{\Omega}_{\mathrm{OHO}}$ is determined: first, by speed $u$ of QHO and, secondly, by spin supercurrent emerging between the same QHO, on the one hand, and the virtual photons created by charged quantum entities constituting the current which, in turn, creates the magnetic field (or by "magnetic" electrons if the magnetic field is created by a permanent magnet), on the other hand. As spin supercurrent is not shielded by electromagnetic screens, the precession of QHO spin may exist in the physical vacuum under shielding of magnetic field, that is at $\mathbf{B}=0(\mathbf{u}=0)$.

For the description of magnetic vector potential let us introduce moment $\mathbf{M}_{\mathrm{QHO}}$ causing the precession of spin of QHOs that constitute the physical vacuum (see Figure 4):

$$
\mathbf{M}_{Q H O}=\boldsymbol{\Omega}_{\mathrm{QHO}} \times \mathbf{S}_{Q H O} .
$$

As $\operatorname{curl} \mathbf{M}_{\mathrm{QHO}} \uparrow \uparrow \boldsymbol{\Omega}_{\mathrm{QHO}}$, then taking into account Equations (15) and (19) and introducing factor of proportionality $b_{s}$, we may write: $\mathbf{B}=\operatorname{curl}\left(b_{s} \mathbf{M}_{Q H O}\right)$. As according to definition of magnetic vector potential $\mathbf{A}, \mathbf{B}=\operatorname{curl} \mathbf{A}$, it may be assumed that:

$$
\mathbf{A}=b_{s} \mathbf{M}_{Q H O} .
$$

Note. Using Equations (16), (19) and (24), magnetic induction $B$ may be expressed as $B=M_{Q H O} \sqrt{4 \pi \rho} \cdot c /\left(\Omega_{Q H O} \cdot S_{Q H O}\right)$.

Let us return to Figure 2 and explain the emergence of interferometer rings at the output of interferometer, that is, explain a change in wave characteristics of quantum entities of beam $C_{2}$ moving in region $\delta$ (in the region of action of field-free vector potential). To this end let us denote the precession frequency of spin of QHO in region $\delta$ as $\left(\Omega_{Q н O}\right)_{\delta}$ and precession frequency of spin of 
virtual photon created by a quantum entity of beam $C_{2}$ as $\left(\omega_{v}\right)_{v p}$ (according to equality (20) $\left(\omega_{v}\right)_{v p}$ is also the wave function frequency of quantum entities).

As a result of action of spin supercurrent between the virtual photon and QHO located in region $\delta$ and according to condition (5), the following takes place:

$$
\left|\left(\Omega_{Q H O}\right)_{\delta}-\left(\omega_{v}\right)_{v p}\right| \geq\left|\left(\Omega_{Q H O}\right)_{\delta}^{\prime}-\left(\omega_{v}\right)_{v p}^{\prime}\right|,
$$

where $\left(\Omega_{Q H O}\right)_{\delta}^{\prime}$ and $\left(\omega_{v}\right)_{v p}^{\prime}$ are the values of respectively $\left(\Omega_{Q H O}\right)_{\delta}$ and $\left(\omega_{v}\right)_{v p}$ after the action of spin supercurrent. As quantum entities of beams $C_{1}$ and $C_{2}$ at the output of the source of quantum entities have equal wave function frequencies, $\left(\omega_{v}\right)_{v p}$, the difference $\Delta\left(\omega_{v}\right)_{v p}$ in those frequencies at the input of interferometer according to inequality (26) is determined as: $\Delta\left(\omega_{v}\right)_{v p}=\left(\omega_{v}\right)_{v p}^{\prime}-\left(\omega_{v}\right)_{v p}$. This difference results in appearance of interference rings.

It should be noted that the characteristics of quantum entities placed in the region of action of magnetic vector potential influence the value of the magnetic vector potential in the region. Let us prove it. According to Equation (26), in general the following holds:

$$
\left(\Omega_{\text {QHO }}\right)_{\delta} \neq\left(\Omega_{Q H O}\right)_{\delta}^{\prime} .
$$

Consequently, according to Equations (24) and (27), at placing of quantum entities in region $\delta$ of the physical vacuum the moment causing the precession of spin of QHO in region $\delta$ will be changed from $\left(\Omega_{\mathrm{QHO}}\right)_{\delta} \times \mathbf{S}_{\mathrm{QHO}}$ to $\left(\Omega_{\mathrm{OHO}}\right)_{\delta}^{\prime} \times \mathbf{S}_{\mathrm{OHO}}$. Thus according to Equation (27) a problem of measurement of magnetic vector potential arises, because the measurement system may influence the value of the magnetic vector potential.

\subsection{Near-Field Antenna Effect}

The explanation of near-field antenna effect as well as of magnetic vector potential effect is based on the properties of the physical vacuum consisting of QHOs characterized by zero-point energy.

The antenna generating electromagnetic oscillations is an oscillating electric dipole [14] [15]. This electric dipole interacts with QHOs located near antenna, like with electric dipoles. As a result of this interaction, the oscillations of electric dipole moment $\left(\mathbf{d}_{\mathrm{OHO}}\right)_{a}$ of QHOs located near antenna arise, that is we have

$$
\frac{\partial\left(\mathbf{d}_{\text {OнО }}\right)_{a}}{\partial t} \neq 0 .
$$

As according to condition (13) $\left(\mathbf{d}_{Q \mathrm{QHO}}\right)_{a} \uparrow \downarrow\left(\mathbf{S}_{Q \mathrm{QHO}}\right)_{a}$, the following takes place in the physical vacuum consisting of QHOs:

$$
\frac{\partial\left(\mathbf{S}_{Q H O}\right)_{a}}{\partial t} \neq 0
$$


where $\left(\mathbf{S}_{\mathrm{QHO}}\right)_{a}$ is spin of QHO located near antenna. The change in $\left(\mathbf{S}_{\mathrm{QHO}}\right)_{a}$ means a change in precession and deflection angles of the precessing spins of QHOs located near antenna. According to Equation (1), spin supercurrent $J$ emerges between QHOs located near antenna, on the one hand, and QHOs located in other regions of physical vacuum, on the other hand. As a result of action of spin supercurrent, the characteristics of spin $\left(\mathbf{S}_{Q H O}\right)_{\text {phys.vac. }}$ of QHOs located in other regions of physical vacuum change, that is, the following takes place in other regions:

$$
\frac{\partial\left(\mathbf{S}_{\text {QHO }}\right)_{\text {phys.vac. }}}{\partial t} \neq 0 .
$$

According to condition (14), the electric field $\left(\mathbf{E}_{Q H O}\right)_{\text {phys.vac. }}$ relates to spin $\left(\mathbf{S}_{\text {Qно }}\right)_{\text {phys.vac. }}$ of QHO, and it follows from Equation (30):

$$
\frac{\partial\left(\mathbf{E}_{\text {QHO }}\right)_{\text {phys.vac. }}}{\partial t} \neq 0 .
$$

Thus, according to Equations (1) and (28)-(31), electric oscillations with characteristics of electric oscillations generated by antenna emerge in the physical vacuum. That is, the following transformations take place in the physical vacuum:

$$
\frac{\partial\left(\mathbf{d}_{\text {QнО }}\right)_{a}}{\partial t}\left(\frac{\partial\left(\mathbf{S}_{\text {QнО }}\right)_{a}}{\partial t}\right) \neq 0 \Rightarrow J \neq 0 \Rightarrow \frac{\partial\left(\mathbf{S}_{\text {QHО }}\right)_{\text {phys.vac. }}}{\partial t}\left(\frac{\partial\left(\mathbf{E}_{\text {Qно }}\right)_{\text {phys.vac. }}}{\partial t}\right) \neq 0 \text { (32) }
$$

These electric oscillations accompany spin supercurrent; consequently, the speed of their spreading in the physical vacuum consisting of QHOs equals the speed of spin supercurrent, which is greater than the speed of light, see Equation (12). It is a little similar to Cherenkov's radiation [27]: the electron moving at a speed greater than the speed of light radiates photons. If to observe only radiated photons, we discover that the speed of motion of photons is greater than the speed of light.

Note. The researchers of near-field antenna effect discover also the oscillation of gravitational field near antenna [31]. Let us discuss this phenomenon on the basis of properties of QHOs.

Due to the action of spin supercurrent in the near antenna region, which equalizes respectively the angles of precession and angles of deflection of precessing spins (Equation (1)), the orientation of QHOs' spins in the same direction in the antenna region may take place (let us denote the total spin of oriented QHOs' spins as $\left(\mathbf{S}_{\mathrm{OHO}}\right)_{\text {total }}$ ). According to condition (13), the orientation of total electric dipole moment $\left(\mathbf{d}_{\text {OHO }}\right)_{\text {total }}$ of the QHOs in the region also takes place:

$$
\left(\mathbf{d}_{\text {OHO }}\right)_{\text {total }} \uparrow \downarrow\left(\mathbf{S}_{\text {OHO }}\right)_{\text {total }} .
$$

In an external nonhomogeneous electric field $\mathbf{E}_{e}$, the force $\mathbf{F}_{Q H O}$ acts on these QHOs. The force $\mathbf{F}_{Q H O}$ is determined [10] as 


$$
\mathbf{F}_{Q H O}=\left(\left(\mathbf{d}_{Q H O}\right)_{\text {total }} \nabla\right) \mathbf{E}_{e}
$$

where $\nabla$ is the nabla operator. In particular, if $\left(\mathbf{S}_{Q H O}\right)_{\text {total }}$ directs towards the Earth, then in the negative electric field of the Earth the force $\mathbf{F}_{Q H O}$ acts oppositely to the gravitation force. As all quantum entities create virtual photons which are vortices in the physical vacuum consisting of QHOs, the force $\mathbf{F}_{Q H O}$ emerging in this vacuum will act on the virtual photons and consequently on the quantum entities creating the virtual photons.

As an example of action of force $\mathbf{F}_{\mathrm{OHO}}$, let us consider the results of experiments on gyroscope's rotations around the vertical axis relative to the Earth [32]. At the right-hand rotation, the decrease in weight of gyroscope took place. The magnitude of decrease in weight did not depend on shielding the gyroscope from external magnetic field $(0.35 \mathrm{G})$. That is, the change in weight was not of magnetic nature. This phenomenon can be accounted for by the emergence of force $\mathbf{F}_{\mathrm{OHO}}$ under the action of the electric field of the Earth. Due to the Barnett effect [33], at rotation of gyroscope the orientation of spins of the QHOs at the location of gyroscope takes place. At the right-hand rotation these spins are pointed downwards, that is towards the Earth. If to take into account that the surface of Earth has a negative charge, then, according to Equations (33) and (34), force $\mathbf{F}_{Q H O}$ acting on QHOs that constitute this vacuum is directed from the Earth, that is, opposite to the vector of gravitation. In the experiments, it may look as a decrease in the weight of the objects in this region of vacuum.

\section{Conclusions}

Quantum correlations between quantum entities may be performed by spin supercurrent emerging between virtual photons (virtual particles pairs) created by those quantum entities.

Magnetic vector potential is proportional to the moment causing the precession of spin of quantum harmonic oscillators (QHOs), which constitute the physical vacuum and are characterized by zero-point energy; in the region of non-zero magnetic field, the frequency of this precession is oriented along the direction of magnetic field. The spin supercurrent emerging between these QHOs and virtual photons (virtual particles pairs) produced by the quantum entities creating magnetic field affects the frequency of this precession.

The near-field antenna effect takes place as a result of action of spin supercurrent between quantum harmonic oscillators (that constitute the physical vacuum) located near antenna, on the one hand, and quantum harmonic oscillators located in other regions, on the other hand. The spin supercurrent gives rise to oscillations of electric field of quantum harmonic oscillators, the latter being electric dipoles. Thus, electric oscillations may spread at the speed of spin supercurrent in the physical vacuum consisting of quantum harmonic oscillators characterized by zero-point energy. As spin supercurrent is an inertia free process, its speed may be greater than the speed of light, which does not contradict postulates of special relativity that set limits to the speed of inertial 
systems only.

(It is a little similar to Cherenkov's radiation: the electron moving at a speed greater than the speed of light in the medium radiates photons. If to observe only radiated photons, we discover that the speed of motion of photons is greater than the speed of light.)

\section{Acknowledgements}

The author is grateful to Mr. Mikhail Boldyrev for his assistance in translating this paper into English.

\section{Conflicts of Interest}

The author declares no conflicts of interest regarding the publication of this paper.

\section{References}

[1] Heisenberg, W. (1927) Zeitschrift für Physik, 43, 172-198. (In German) https://doi.org/10.1007/BF01397280

[2] Schrödinger, E. (1936) Mathematical Proceedings of the Cambridge Philosophical Society, 32, 446-452.

[3] Wichmann, E.H. (1971) Quantum Physics. Berkeley Physics Course, McGraw-Hill Book Company, New York.

[4] Klyshko, D.N. (1994) Physics Uspekhi, 37, 1097-1122. https://doi.org/10.1070/PU1994v037n11ABEH000054

[5] Belinskii, A.V. (2003) Physics Uspekhi, 46, 877-881. https://doi.org/10.1070/PU2003v046n08ABEH001393

[6] Tittel, W., Brendel, J., Gisin, B., et al. (1998) Physical Review A, 57, 3229. https://doi.org/10.1103/PhysRevA.57.3229

[7] Scarani, V., Tittel, W., Zbinden, H. and Gisin, N. (2000) Physics Letters A, 276, 1-7. https://doi.org/10.1016/S0375-9601(00)00609-5

[8] Born, M. (1962) Einstein's Theory of Relativity. Dover Publications, New York.

[9] Einstein, A., Podolsky, B. and Rosen, N. (1935) Physical Review, 47, 777. https://doi.org/10.1103/PhysRev.47.777

[10] Purcell, E.M. (1965) Electricity and Magnetism. Berkeley Physics Course, Vol. 2, McGraw-Hill Book Company, New York.

[11] Ehrenberg, W. and Siday, R.E. (1949) Proceedings of the Physical Society (London) $B, 62,8-21$.

[12] Aharonov, Y. and Bohm, D. (1959) Physical Review, 115, 485-491. https://doi.org/10.1103/PhysRev.115.485

[13] Chambers, G. (1960) Physical Review Letters, 5, 3. https://doi.org/10.1103/PhysRevLett.5.3

[14] Walker, W.D. (1999) Superluminal Near-Field Dipole Electromagnetic Fields. International Workshop "Lorentz Group CPT and Neutrinos" Zacatecas, Mexico, 23-26 June 1999, 16 p.

[15] Walker, W.D. (2000) Experimental Evidence of Near-Field Superluminally Propagating Electromagnetic Fields. In: Amoroso, R.L., et al., Eds., Gravitation and Cos- 
mology: From the Hubble Radius to the Planck Scale, Kluwer Academic Publishers, Printed in Netherlands, 189-196.

[16] Borovic-Romanov, A.S., Bunkov, Yu.M., Dmitriev, V.V., Mukharskii, Yu.M. and Sergatskov, D.A. (1989) Physical Review Letters, 62, 1631.

https://doi.org/10.1103/PhysRevLett.62.1631

[17] Bunkov, Yu.M. (2009) Journal of Physics. Condensed Matter, 21, Article ID: 164201. https://doi.org/10.1088/0953-8984/21/16/164201

[18] Dmitriev, V.V. and Fomin, I.A. (2009) Journal of Physics: Condensed Matter, 21, Article ID: 164202. https://doi.org/10.1088/0953-8984/21/16/164202

[19] Puthoff, H.E. (1989) Physical Review A, 40, 4857-4862. https://doi.org/10.1103/PhysRevA.40.4857

[20] Einstein, A. and Stern, O. (1913) Annelen der Physik, 345, 551-560. https://doi.org/10.1002/andp.19133450309

[21] Milonni, P.W. (1994) The Quantum Vacuum. Academic Press, Inc., Harcourt Brace \& Company Publishers, Cambridge, MA.

[22] Weber, M.H. and Lynn, K.G. (2000) Radiation Physics and Chemistry, 58, 749-753. https://doi.org/10.1016/S0969-806X(00)00252-8

[23] Doctorovitch, Z.I. (2005) Engineering \& Automation, No. 4, 115-118. (In Russian)

[24] Boldyreva, L.B. (2018) International Journal of Physics, 6, 128-138.

[25] Boldyreva, L.B. (2019) Journal of Modern Physics, 10, 20-34.

[26] Sedov, L.I, (1971-1972) A Course in Continuum Mechanics. Wolters-Noordhoff, Groningen, 1-4.

[27] Čerenkov, P.A. (1937) Physical Review, 52, 378. https://doi.org/10.1103/PhysRev.52.378

[28] Boldyreva, L.B. (2017) International Journal of Physics, 5, 141-146. http://pubs.sciepub.com/ijp/5/4/6/

[29] Boldyreva, L.B. and Sotina, N.B. (1992) Physics Essays, 5, 510-513.

[30] Boldyreva, L.B. (2014) International Journal of Quantum Information, 12, Article ID: 1450007. https://doi.org/10.1142/S0219749914500075

[31] Walker, W.D. and Dual, J. (1998) Propagation Speed of Longitudinally Oscillating Gravitational and Electrical Fields. https://arxiv.org/abs/gr-qc/9706082v2

[32] Hayasaka, H. and Takeuchi, S. (1989) Physical Review Letters, 63, 2701-2704. https://doi.org/10.1103/PhysRevLett.63.2701

[33] Barnett, S.J. (1915) Physical Review, 6, 239-270. 